Pädiatrie und Pädologie 2014 · 49:3

DOI 10.1007/s00608-014-0163-1

๑) Springer-Verlag Wien 2014
Franz Waldhauser

AG Schwerpunksetzung in der „Politische Kindermedizin“, Wien

\title{
Kompetenzzentren und Versorgungsnetzwerke
}

Die Behandlung von Kindern und Jugendlichen mit seltenen, komplexen und diagnostisch / therapeutisch aufwendigen Erkrankungen erfordert besondere Einrichtungen, um für diese Patienten ein optimales Therapieresultat zu erlangen. Zahlreiche Outcome-Studien belegen, dass die therapeutischen Ergebnisse in spezialisierten Einrichtungen bei weitem besser sind als in Einrichtungen mit generalisiertem Versorgungsauftrag und wenig spezifischer Erfahrung.

Unisono fordern internationale Experten Kompetenz-Zentren, welche die notwendigen personellen und maschinellen Einrichtungen vorhalten und mindestens eine bestimmte Anzahl von Patienten pro Jahr betreuen, um auch Erfahrung zu sammeln. Denn auch in der Medizin gilt: Übung macht den Meister. Erst wenn diese Strukturqualität gegeben ist, sollte eine Therapie angeboten werden dürfen. Schließlich sind die Ergebnisse transparent, für den nationalen und internationalen Vergleich, offen zu legen. Denn ein Anspruch auf Kompetenz sollte auch zweifelsfrei belegt werden. Dieses Recht haben die Patienten, die Gesundheitspolitiker/ -manager und die finanzierende Bevölkerung.

\section{Was ist schon umgesetzt?}

Weltweit ist das Problem erkannt, und weltweit wird nach idealen Strukturierungen gesucht, um den Bedürfnissen dieser besonders benachteiligten Patienten gerecht zu werden.

In Staaten mit dezentraler Patientenversorgung - wie den deutschsprachigen Ländern - stellt dies besondere Anforderungen an die verantwortlichen Gesundheitspolitiker und -manager.
Unter den deutschsprachigen Ländern hat sich die Schweiz dieser Herausforderung als erstes offensiv gestellt: Mit der Interkantonalen Vereinbarung zur Hochspezialisierten Medizin (HSM) 2009 hat sie für das gesamte Staatsgebiet ein Gremium geschaffen, das verbindliche Strukturen zur Betreuung der Patienten mit seltenen, komplexen Erkrankungen implementieren kann. Tatsächlich wurden bisher für 22 Fachbereiche zeitlich limitierte Entscheidungen gefällt (Details siehe im nachfolgenden Artikel, Seite 20).

In Deutschland hat man zunächst mit der Einführung von verbindlichen Mindestmengen ein ähnliches Ziel verfolgt. Vor allem im operativen Bereich, aber nicht nur dort, wurde vereinbart, dass eine Krankenanstalt mindestens eine bestimmte Zahl von Behandlungen pro Jahr in einem gewissen Bereich durchführen muss, ansonsten sind diese Behandlungen untersagt und werden nicht vergütet. Zurzeit gelten 20 Lebertransplantationen, 10 komplexe Ösophagus-, 10 komplexe Pankreasinterventionen, 25 Stammzelltransplantationen und 14 sehr kleine Frühgeborene $(<1.250 \mathrm{~g})$ pro Jahr als Mindestmengen für die jeweiligen Kompetenz-Zentren.

Durch die Veröffentlichung des Aktionsplans für Menschen mit Seltenen Erkrankungen des deutschen BM für Gesundheit, BM für Bildung und Forschung und der Allianz Chronischer Seltener Erkrankungen im April 2013 wurde ein weiterer Schritt zur Implementierung von Spezialeinrichtungen für diese besonders benachteiligte Gruppe von Patienten in Deutschland getan.

Die Österreichische Bundesregierung bekennt sich in ihrem Regierungsprogramm von 2013 zu einer Verbesserung des
„Zugangs der PatientInnen zur Spitzenmedizin....mittels überregionaler Leistungsangebotsplanung für hochspezialisierte $\mathrm{Me}$ dizin“. Das BM f. Gesundheit war federführend bei der Beauftragung der Gesundheit Österreich $\mathrm{GmbH}$, Österreichs nationalem Gesundheitsplaner, einen nationalen Aktionsplan für seltene Erkrankungen (NAPse)zukonzipieren.Die diesbezüglichen Arbeiten sind weit fortgeschritten und stehen vor dem Abschluss.

Über Initiative der AG Schwerpunktsetzung in der Politischen Kindermedizin haben die European Paediatric Association, die Gesundheit Österreich GmbH, der Hauptverband der Sozialversicherungsträger, und der Betroffenenverein „Pro rare“ für 6. und 7. November 2014 in Salzburg ein Joint-Meeting vereinbart. Das Meeting thematisiert theoretische und praktische Fragen im Zusammenhang mit Kompetenzzentren und ihrer Vernetzung, erste Erfahrungen im deutschsprachigen Ausland mit derartigen Einrichtungen und potentielle Fallstricke bei der Implementierung solcher Strukturelemente in Österreich (siehe Seite 5 und http://www.polkm.org)

Liebe Leserin, lieber Leser, lassen Sie sich diese Veranstaltung nicht entgehen! Registrieren Sie das dargestellte „Meeting“, gestalten Sie bei der Strukturierung der Einrichtungen für diese besonders benachteiligten Patient mit! Denn, wer zu spät kommt, den straft die Geschichte.

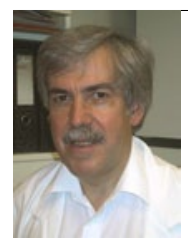

a.o.Univ.-Prof. Dr. Franz Waldhauser AG Schwerpunktsetzung in der „Politischen Kindermedizin" www.polkm.org f.waldhauser@aon.at 